\title{
A Study on an Energy Conservation and Interconnection Scheme between WSN and Internet Based on the 6LoWPAN
}

\author{
Runtong Zhang, ${ }^{1}$ Fuzhi Chu, ${ }^{1}$ Quan Yuan, ${ }^{1}$ and Wei Dai ${ }^{2}$ \\ ${ }^{1}$ Beijing Jiaotong University, Beijing 100044, China \\ ${ }^{2}$ Victoria University, Melbourne, VIC 8001, Australia \\ Correspondence should be addressed to Quan Yuan; yuanquan721@hotmail.com
}

Received 14 October 2013; Accepted 24 February 2014

Academic Editor: David Taniar

Copyright (c) 2015 Runtong Zhang et al. This is an open access article distributed under the Creative Commons Attribution License, which permits unrestricted use, distribution, and reproduction in any medium, provided the original work is properly cited.

\begin{abstract}
Wireless sensor network (WSN), which has broad application prospects, consists of small nodes with sensing, computation, and communications capabilities. IPv6 is used over low power WPAN (wireless personal area network) which is run by 6LoWPAN technology on the LoWPAN devices. In order to establish seamless connection of two heterogeneous networks, that is, WSN and IPv6 network, this paper proposes an improved energy conservation and interconnection scheme (ECIS) based on the analysis of the current schemes. Detailed design of each functional component in the new scheme is proposed, and an expansion of the SSCS module in NS2 802.15.4 simulator is examined. Comprehensive experiments on the simulation platform show that the newly proposed scheme is advantageous over existing results.
\end{abstract}

\section{Introduction}

Wireless sensor network (WSN) is an integrated intelligent information system including information collection, information transmission, and information processing, which has broad application prospects [1]. IPv6 over low-power WPAN $(6 \mathrm{LoWPAN})[2,3]$ is a kind of network architecture for transmitting IPv6 packets based on IEEE 802.15.4 standard. The concept was born from the idea that the Internet protocol could and should be applied to even the smallest of devices. The IPv6 technology can be used to build a sensor network. The PHY and MAC layers provided by IEEE 802.15.4 are used in 6LoWPAN while the underlying technology and network layer uses the IPv6 protocol.

With the in-depth development of IPv6 network along with its extensive applications and sensor network technology, it becomes a significant area for us to study the combination of the key technology from IPv6 and WSN, which will bring great changes to people's lives and work. It also has an important value for the development and application of Internet of Things by building a low-cost and high-efficiency dual-network interconnection. Sponsored by Beijing Natural Science Fund, this research proposes a new improved energy conservation and interconnection scheme (ECIS) between the dual-networks in order to improve energy efficiency and reducing energy consumption by studying the interconnection technique between WSN and Internet as well as 6LoWPAN technology.

\section{Related Works}

2.1. Network Analysis of Energy Consumption. As Internet traffic increases, its power utilization grows. This growth in energy consumption must be considered seriously in any future Internet design; otherwise, it may constrain the growth of the Internet itself.

The subject of improving energy efficiency and reducing energy waste was first raised by the computer semiconductor industry. Intel's 486-DX processor was the first device which can support power management. The first official ACP standards were promulgated in 1996 [4]. As time goes by, more and more energy-saving mechanisms and enhanced hardware were created so that most of the CPUs could achieve higher performance using less power.

Most of the network devices are derived from the development of computer technology. Ad hoc hardware engine [5] and silicon chips simplify the complex time demanding traffic operation. In the field of research and industrial development, 
the introduction of energy-saving technologies and standards for a particular network can distinguish many network devices' intrinsic differences in hardware and provides an alternative solution.

Overall, most of the research results are from the power saving mechanisms of computer system and standards of power management, which can be divided into the following three categories.

2.1.1. Redesign. Redesign means to introduce and design a network equipment component which has higher energy efficiency to optimize the structure and reduce complexity of the devices.

The most challenging solution is the use of pure optical switching fabric [6]. It examines the potential of photonic switching to reduce energy consumption by determining the contribution of cross connects and buffers to the total energy consumption of the Internet. After estimating the power consumption of the functional blocks in the network, it shows that the power consumption of the switch fabrics as a percentage of the total power consumption rises slowly with increasing access rate. At an access rate of $100 \mathrm{Mb} / \mathrm{s}$, the switch fabrics and buffers consume approximately $7 \%$ and $2 \%$, respectively, of the total energy consumption of the Internet. The research concludes that even if photonic switching and buffering technologies provide lower power solutions than electronics, photonic technologies alone will not solve the looming "energy bottleneck" problem [7].

Roberts [8] focuses on a simple yet powerful idea: if a router can identify the first packet in a flow, it can just prescreen the remaining packets and bypass the routing and queuing stages. This approach would boost throughput, reduce packet loss and delays, allow new capabilities like fairness controls, and save power, size, and cost. The approach is called flow management.

Baldi and Ofek [9] show how to "trade" global time for electricity utilized by the Internet. The time-based IP switching approach realized by pipeline forwarding, and specifically time-driven switching (TDS)/fractional lambda switching (F $\lambda \mathrm{S})$, recently demonstrated by our novel test bed implementation significantly reduced the electricity requirement. The strength of F $\lambda \mathrm{S} / \mathrm{TDS}$ is its simplicity, while not compromising the three most desired properties for the future "green" Internet.

Most researches on router power management are at component level or link level, treating routers as isolated devices. A complementary approach is to facilitate power management at network level by routing traffic through different paths to adjust the workload on individual routers or links. Given the high path redundancy and low link utilization in today's large networks, research by Zhang et al. [10] could potentially allow more network devices or components to go into power saving mode. They propose an intradomain traffic engineering mechanism, GreenTE, which maximizes the number of links that can be put into sleep mode under given performance constraints such as link utilization and packet delay. Using network topologies and traffic data from several wide area networks, their evaluation shows that GreenTE can reduce line-cards' power consumption by $27 \%$ to $42 \%$ under constraints that the maximum link utilization is below $50 \%$ and the network diameter remains the same as in the shortest path routing.

Chiaraviglio and his colleagues [11] consider a real IP backbone network and a real traffic profile. They evaluate the energy cost of running it, and, speculating on the possibility of selectively turning off spare devices whose capacity is not required to transport off-peak traffic, they show that it is possible to easily achieve more than $23 \%$ of energy saving per year, that is, to save about $3 \mathrm{GWh} /$ year considering today's power footprint of real network devices.

2.1.2. Dynamic Adaptive Mechanism. Dynamic adaptive mechanism mainly refers to the dynamic adaptive network equipment and resources. It automatically adjusts the packet processing engine and the capacity of the network interface to satisfy the actual traffic loads and requirements. This function can be illustrated through dynamic voltage scaling and idle logic that are allowed to dynamically balance the relationship between packet data service performance and energy loss.

Currently the most important part of the network equipment does not include this hardware feature [12,13], but it is now as one of the processor power management features and is also used in other key areas of rapid development of hardware technology. Noguera and Kennedy [13] present the design and evaluation of two forms of power management schemes that reduce the energy consumption of networks. The first is based on putting network components to sleep during idle times, reducing energy consumed in the absence of packets. The second is based on adapting the rate of network operation to the committed workload, reducing the energy consumed when actively processing packets. The research shows that these savings approach the maximum level achievable by any algorithms using the same power management primitives. Moreover, this energy can be saved without noticeably increasing loss and with a small and controlled increase in latency $(<10 \mathrm{~ms})$. Finally, the research shows that both the sleeping and rate adaptation are valuable depending (primarily) on the power profile of the network equipment and the utilization of the network itself.

Gupta and Singh [14] examine the somewhat controversial subject of energy consumption of networking devices in the Internet, motivated by data collected by the United States Department of Commerce. They discuss the impact on network protocols of saving energy by putting network interfaces and other router and switch components to sleep. Using sample packet traces, they show that it is indeed reasonable to do this and then discuss the changes that may need to be made to current Internet protocols in order to support a more aggressive strategy for sleeping. The impact of saving energy is huge, particularly in the developing world where energy is a precious resource whose scarcity hinders widespread Internet deployment.

Cianfrani et al. [15] analyze the challenging problem of energy saving in IP networks. A novel network-level strategy based on a modification of current link-state routing protocols, such as OSPF, is proposed. According to this strategy, IP routers are able to power off some network links during low traffic periods. The proposed solution is a three-phase 
algorithm: in the first phase some routers are elected as exporter of their own shortest path trees (SPTs); in the second one the neighbors of these routers perform a modified Dijkstra algorithm to detect links to power-off; in the last one new network paths on a modified network topology are computed. Performance study shows that, in an actual IP network, even more than the $60 \%$ of links can be switched off.

Ricciardi et al. [16] envision the necessity of introducing energy efficiency and energy awareness in the design, configuration, and management of networks and specifically in the design and implementation of enhanced control-plane protocols to be used in next generation networks.

2.1.3. Sleep Mechanisms. The method is used for intelligent sleeping mechanism, optionally changing the network or device that is not used under the low-power standby mode to wake up again when necessary. However, due to the current network design, services and applications that run continuously and have ready access mode and standby mode must show support for a particular node agent technology to maintain sleep and components of the "continuous operation."

Christensen and Gunaratne propose methods for reducing energy consumption of networked desktop computers. Using traffic characterization of university dormitory computers, they show that there is significant idle time that can be exploited for power management. However, current Ethernet adapters in desktop computers lack the capabilities needed to allow existing system power management features to be enabled. They address this problem with a proxying Ethernet adapter that handles routine network tasks for a desktop computer when it is in a low-power sleep mode. The proxying adapter can allow existing power management features in desktop computers to remain enabled and have the computer be "on the network" at all times. The energy that can be saved under such an approach is in the range of $0.8-2.7$ billion US dollars/year.

\subsection{WSN and Internet}

2.2.1. Connectivity Way between WSN and Internet. Now there are three ways to make WSN access the Internet including peer to peer, overlapping manner and WSN over TCP/ IP.

(a) Peer to Peer. According to the different protocol layer where gateway nodes work, it can be further divided into the application gateway and NAT (network address translation) gateway. Application gateway is the simplest way to realize the interconnection, which sets up one or more proxy servers between internal and external networks. NAT gateway transforms the address and protocol between WSN and network in network layer in this situation that intranet uses the private protocol treating address as the center [17].

(b) Overlap Method. Overlap method is an interconnection through protocol bearing instead of protocol conversion under the condition that the protocol stacks of WSN and IP network are different. There are two ways including WSN

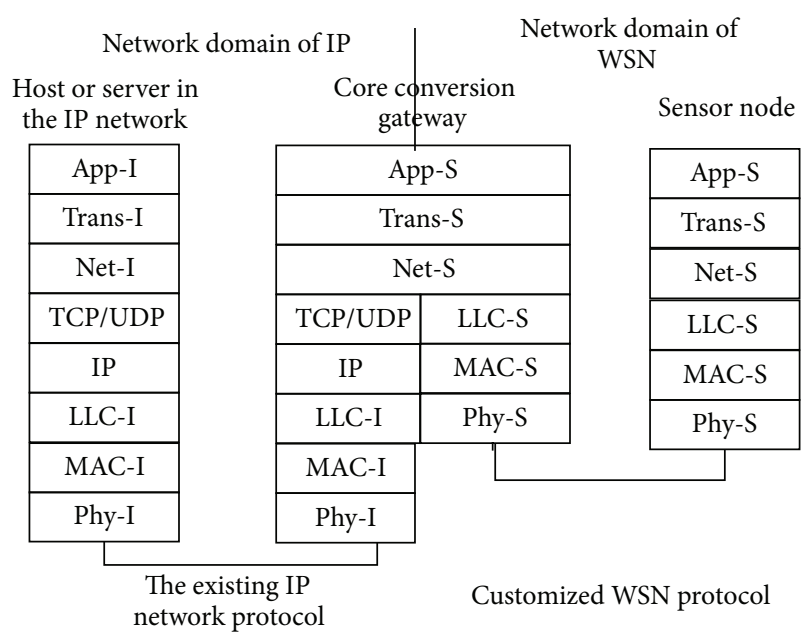

FIGURE 1: WSN over TCP/IP.

Network domain of IP Network domain of WSN

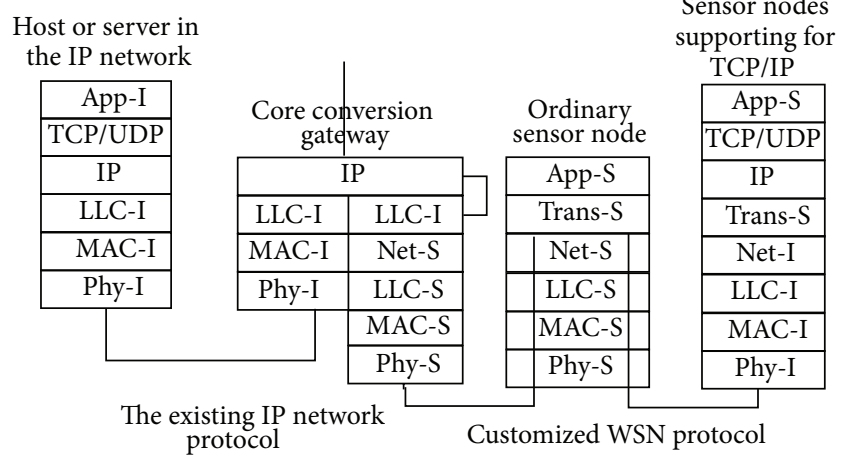

Figure 2: IPv6 over WSN.

over IP and IP over WSN. The first one is similar to VPN (virtual private network) which makes a network connection of private network in IP as shown in Figure 1. In real network, each WSN node runs a private protocol which is adapted to WSN characteristics. In virtual network, the network layer of WSN private protocol is treated in the same way when applications based on the TCP/UDP/IP transmit data among virtual nodes in the form of a tunnel [18].

The main part of WSN in IPv6 over WSN still uses a private communications protocol. IPv6 protocol is only used in some special nodes shown in Figure 2. In general, it is not recommended that every ordinary WSN node should support IPv6 protocol.

(c) All-IP. WSN must allow certain nodes to realize protocol conversion or protocol bearing between internal and external networks. In order to make it easier to achieve the WSN and IPv6 network connectivity, All-IP [19] was proposed in recent years. This is a seamless combination between WSN and IPv6, which establishes the network interconnection by ordering each ordinary sensor nodes in WSN $[20,21]$ to support IPv6 protocol. 


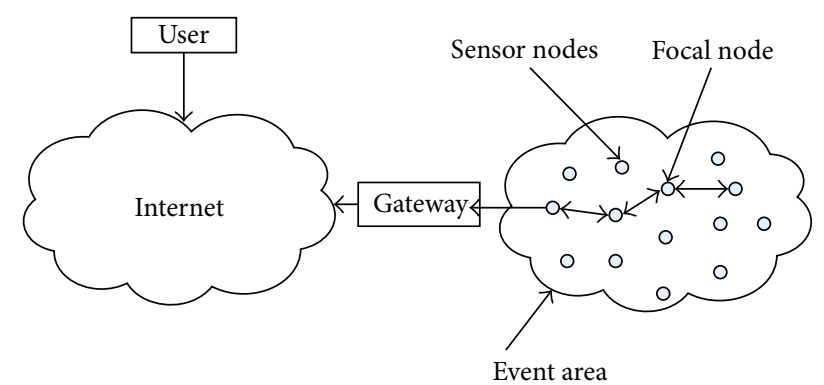

FIGURE 3: Isomorphic network interconnection using a single gateway.

Some studies support the All-IP solution. Some consider that it is the simplest and most convenient way to realize WSN and IPv6 network interconnection by using an Ethernet cable or wireless link like GPRS. There are also other researchers against the all-IP arguing that it is unfeasible because of the different work mechanism, as IP is address centered and WSN is data-centric. It is therefore not clear to achieve IPv6 protocol stack in the WSN nodes.

2.2.2. Connectivity Structure of WSN and Internet. WSN and Internet interconnection structure design is crucial. At present, there are two structures for the sensor network and Internet connectivity [22].

One interconnection structure shown in Figure 3 describes a gateway as an interface for the sensor network and Internet connectivity. This structure is called isomorphic Internetworking for all the nodes have the same functionality except for the gateway. It uses the gateway to shield sensor networks and to provide real-time information services and interoperability function for remote Internet users. Isomorphic network interconnection structure puts Internet connected interface in the outside gateway of WSN.

The advantage of this structure is that the intermediate steps can be implemented between the sensor network and the Internet. It facilitates a smooth docking of the two data streams, making it easy to manage. The disadvantage is that large streams of data streams gather in around the nodes near gateway. The nodes near gateway have excessive energy consumption and the distribution of net consumption is uneven, which reduce the survival time of sensor network.

The improvement program is to use multiple gateways as shown in Figure 4 to promote distribution of energy consumption within the network uniform. Either single gateway or multigateway program will result in a certain degree of information redundancy.

The other structure gives IP address to part of the nodes as Internet connectivity interface as shown in Figure 5. These interface nodes can achieve TCP/IP protocol for Internet and specific transport protocol of the sensor network [17]. This structure is called heterogeneous network interconnection. It uses the specific node to shield sensor networks and provide real-time information services and interoperability function for remote Internet users. Compared with the homogeneous

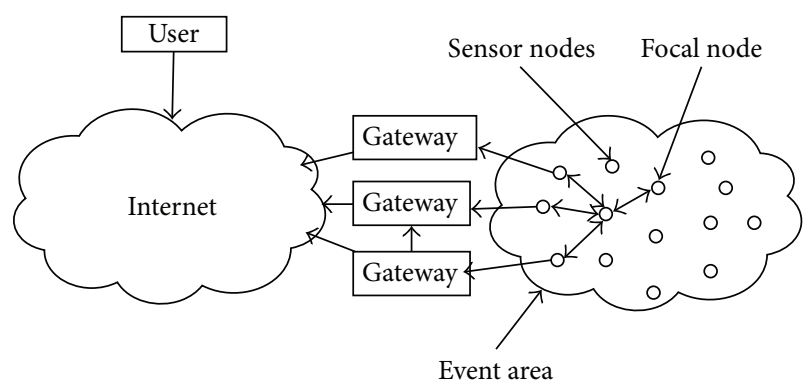

FIGURE 4: Isomorphic network interconnection using multiple gateways.

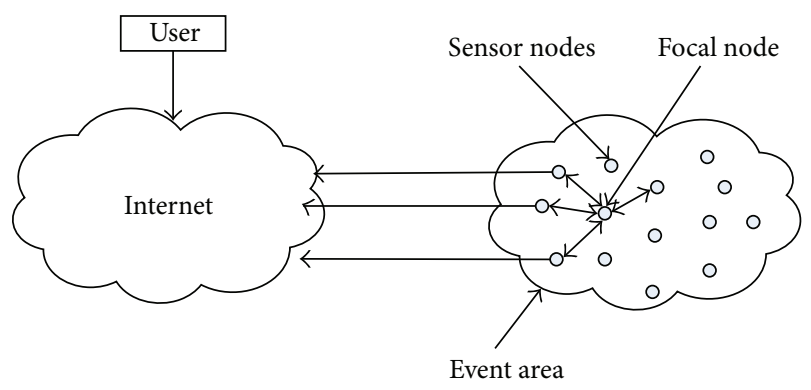

FIGURE 5: Heterogeneous network interconnection of interface node.

network interconnect, the heterogeneous network interconnect has a more uniform distribution of energy consumption and a better data streams integration within the sensor network. It reduces information redundancy. Heterogeneous network interconnection requires a larger extent to adjust the sensor network routing and transmission protocol, increasing the complexity of the sensor network design and management.

2.2.3. Connectivity Problems between WSN and Internet. IPv6 protocol [23], after all, is not a specific protocol for the sensor network communication; hence, there are still a series of problems to be solved in IPv6-based sensor network implementation process.

(a) Multihop Wireless Network. It is very necessary that some or all of the nodes in the sensor network hold the routing forwarding capability. Therefore, for each ordinary node, it is an important issue worthy of further study that it only supports IPv6 protocol on the host side or supports the host side and the router side of IPv6 at the same time. The problem is closely related to the network structure of the sensor network addressing modes and other factors, which needs to combine specific networking mode and take into account machine production considerations.

(b) IPv6 Header Compression. General sensor networks have a very small amount of communication services and very low data rates $(1.100 \mathrm{Kbit} / \mathrm{s})$, which leads to use of standard IPv6 encapsulation format bound to bring heavy packet header overhead. Utilizing a certain way to compress some fields of 
the IPv6, TCP and UDP header is an important way to reduce the header overhead. The current work in this area has made some progress, but in-depth study on the integrated sensor network characteristics of header compression remains to be done.

(c) IPv6 Address Autoconfiguration. Address autoconfiguration is an important technical feature of IPv6. IPv6 supports not only state address autoconfiguration but also stateless address configuration. However, there are also some problems in the existing IPv6 address autoconfiguration in sensor networks. For instance, the central control features brought in many control messages overhead and generated IPv6 address based on the MAC address routing. However, addressing among sensor nodes did not bring any convenience.

(d) Data-Centric Business. To host data-centric business based on the attribute data query, data return process aggregation or fusion is an important feature for many sensor networks that are different from traditional IP network. Therefore, in an address-centric network architecture such as the IPv6, how to support data-centric business effectively, and for the IPv6 based sensor network to resolve access problems, remain to be critical [24].

\section{ECIS between WSN and Internet Based on the 6LoWPAN}

Different from the general computer network, the nodes of the 6LoWPAN network are always based on the battery power. As the state-of-the-art technology, the capacity of a battery is reaching its limit and will be difficult to significantly improve. Replacing the battery is unrealistic in many applications such as military applications. We need to reduce the node energy consumption as far as possible by energy-saving mechanism, which can effectively extend node's working time and overall life of network. According to the characteristics of network, it is one of the most important 6LoWPAN contents that design new protocols, algorithms, and energy-saving strategies to reduce the network energy consumption.

3.1. Energy Control Technology of 6LoWPAN. Compared with the traditional network, 6LoWPAN nodes are generally battery-powered. They always work under the unattended environment for long time. For example, a node with two dry cells has the power about $2200 \mathrm{mAh}$ under the condition of $3 \mathrm{~V}$. If it needs to work for nine months, each node can only have $8.148 \mathrm{mAh}$ power per day. Thus, energy problem is one of the key issues of 6LoWPAN network. In order to deal with the energy-saving problem, the following three aspects should be mainly considered: energy supply, energy consumption, and energy management. Energy supply should mainly cover 6LoWPAN nodes of power supply equipment such as batteries. Energy consumption requires full consideration of the function of 6LoWPAN nodes modules. Energy regulators include energy monitoring and power control.

The energy resources of 6LoWPAN networks are limited. In order to extend life of the system, three aspects including

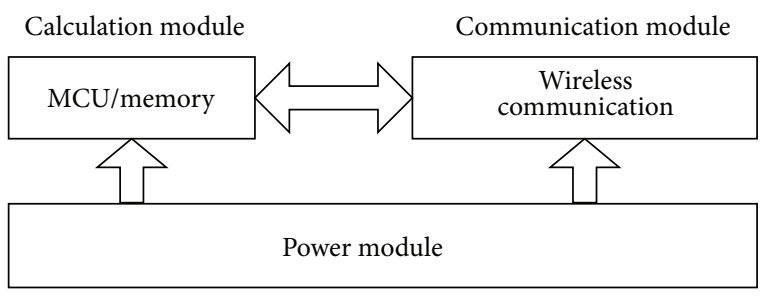

FIGURE 6: Unit module of 6LoWPAN network node.

processor subsystem, energy subsystem, and communication subsystem need to be taken into account. As shown in Figure 6, modular 6LoWPAN network node consists of three parts: calculation module, communication module, and power module.

Calculation module including the microcontroller unit (MCU) and memory is responsible for controlling the operation of 6LoWPAN nodes, computing its own data and other data. For power management, MCU has a number of operation modes such as active, idle, and sleep. Each mode has its own power consumption. The communication module is responsible for performing wireless communication, exchanging control messages, and send-receiving data with other 6LoWPAN nodes. This is the largest part of node energy consumption. Wireless transceiver circuit can work in four conditions including sending, receiving, idle, and dormancy. Apart from the dormant state, energy consumption of other three states is very large. Idle power consumption is close to receiving state. Therefore, when wireless transceiver circuit is in the idle state, it should be placed in a dormant state as long as possible.

The interface control and management of power supply module and calculation modules also have a certain impact on the energy management. For example, adopting threewire switch, reducing the output current, and cutting the power frequently can increase the system life. According to the characteristics of chemical battery, continuous consumption of large current in a long time will make the battery failure for the time being, but then it will gradually return to work.

On the 6LoWPAN nodes, energy consumption of communication module is the largest one, followed by calculation module. Other types of energy consumption are far less than these two. So the focus of 6LoWPAN saving energy is on the communication module and computing module.

3.2. The 6LoWPAN Network Energy-Saving System Design. Based on the feature of 6LoWPAN nodes' modular power consumption nodes, the research of energy control is mainly concentrated on the dormancy mechanism, data fusion to avoid conflict and error correction, and so forth. They are applied in all aspects of calculation module and communication module which should be fully considered. Consequently combining with all the necessary characteristics, the specific design is described in Figure 7.

(a) Energy Saving of Hardware. Low-power consumption components should be adopted in the hardware selection. 


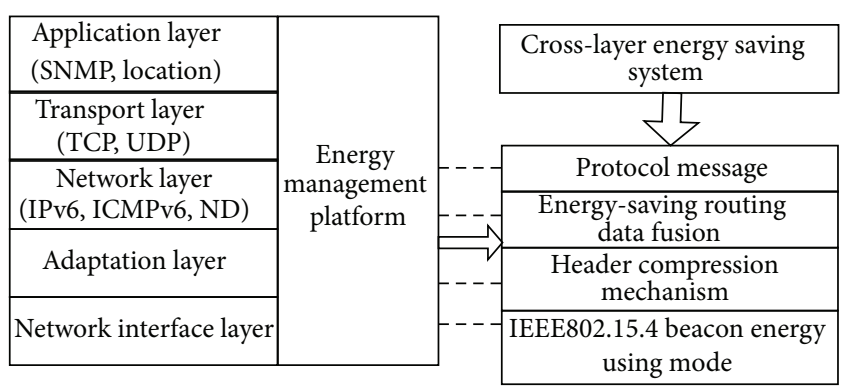

FIGURE 7: Cross-layer energy-saving system.

Trade-off between performance and power consumption reduces power consumption of the whole system. The processor should be capable to adjust working voltage and working frequency and to provide the necessary conditions for dormancy of processing modules. At the same time, energy consumption of wireless transceiver is attributed to its working condition.

(b) Operating System. Operating system can get all the performance requirements of applications and directly control underlying hardware resources in order to make necessary trade-off between performance and energy consumption control. Therefore, dynamic power management and dynamic voltage scaling can effectively save energy and prolong survival time in the operating system.

(c) Dormancy Mechanism. When the node computing and communications modules are idle, these components should be turned off or transferred to a lower-power state that is called dormant state to prolong the lifetime of sensor nodes. However, the transformation between dormant state and working state needs to consume certain amount of energy and have time delay; therefore, state transition strategy for dormancy mechanism is very important.

(d) Network Interface Layer. Network interface layer adopts IEEE802.15.4 protocol that includes two modes: synchronous mode and asynchronous mode. The synchronization mode provides support to dormancy mechanism. A reasonable dormancy mechanism is needed to effectively reduce node power consumption.

(e) Data Fusion. Data processing power consumption is usually less than communication power consumption. For example, the energy is available for computing 3000 instructions when sensor node transmits 1 bit to 100 meters by wireless. Data fusion, redundancy removal, and useful information transmission in the sensor network of 6LoWPAN can achieve the goal of energy saving by reducing traffic effectively.

(f) Link-Layer Optimization. Link-layer optimization includes collision avoidance and error correction. Retransmission caused by conflict results is a great waste of energy. Reducing conflicts can save energy effectively. Error detection and correction mechanisms can also reduce retransmission of packets, which can reduce energy consumption under the condition of a given bit error rate.

(g) Energy-Saving Routing. Based on the common routing protocol, energy-saving routing introduces new measures related to power consumption in order to save energy after considering energy consumption factors. Node energy and data fusion should be considered during the routing protocol design.

Microprocessor is also called the microcontrol unit (MCU). It is mainly used to complete coordination of the system, run communication protocol, collect sensor data, and control other applications. It usually contains a variety of work modes. Generally, processor's power consumption is much less than the counter part for wireless communication. The power consumption of MCU that chooses CMOS technology is proportional to switching frequency. It is also proportional to equivalent capacitance of switch and square of device supply voltage amplitude. The relationship is shown in formula (1). In the formula, $P$ represents the work power. $C$, $V$, and $f$ represent the equivalent capacitance of the switch, the device supply voltage, and operating frequency of chip. The formula shows us that reducing the supply voltage can effectively reduce power consumption. Operating voltage and operating frequency can be adjusted through the software automatically depending on the environment:

$$
P=C * V^{2} * f \text {. }
$$

(h) Topology Management. The purpose of 6LoWPAN network topology management is to ensure that there are active "right" nodes in the network to make the rest of the nodes that are not participating in data transmitting into dormant state. Topology management should, as far as possible, distribute data tasks evenly to all nodes in order to save energy.

(i) Packet Control. Network protocol needs control packets and header to maintain its normal operation. But control packets and header are not required by users. Reducing the number of control packets should be considered during network protocol design. At the same time, length of header can be reduced by header compression mechanism to reduce traffic and achieve the goal of reducing energy consumption.

3.3. Interface Design. The program storage areas and data storage areas of ordinary nodes are so limited that they could not configure the complete network protocol stack and largescale routing protocol. Therefore, we use a hardware gateway device that has rich resources to achieve the interoperability of IPv6-WSN with existing network as shown in Figure 8.

Network protocol stack, which is deployed by IPv6-WSN equipment, gathers monitoring data through a wireless sensor network under routing protocols to the gateway device. After data forwarding processing, gateway device makes access to the various bearer networks to send information to server data system. Upon processing and analyzing, the server data system stores monitoring information in the database. User terminal accesses server data system through existing network protocols and obtains IPv6-WSN devices 


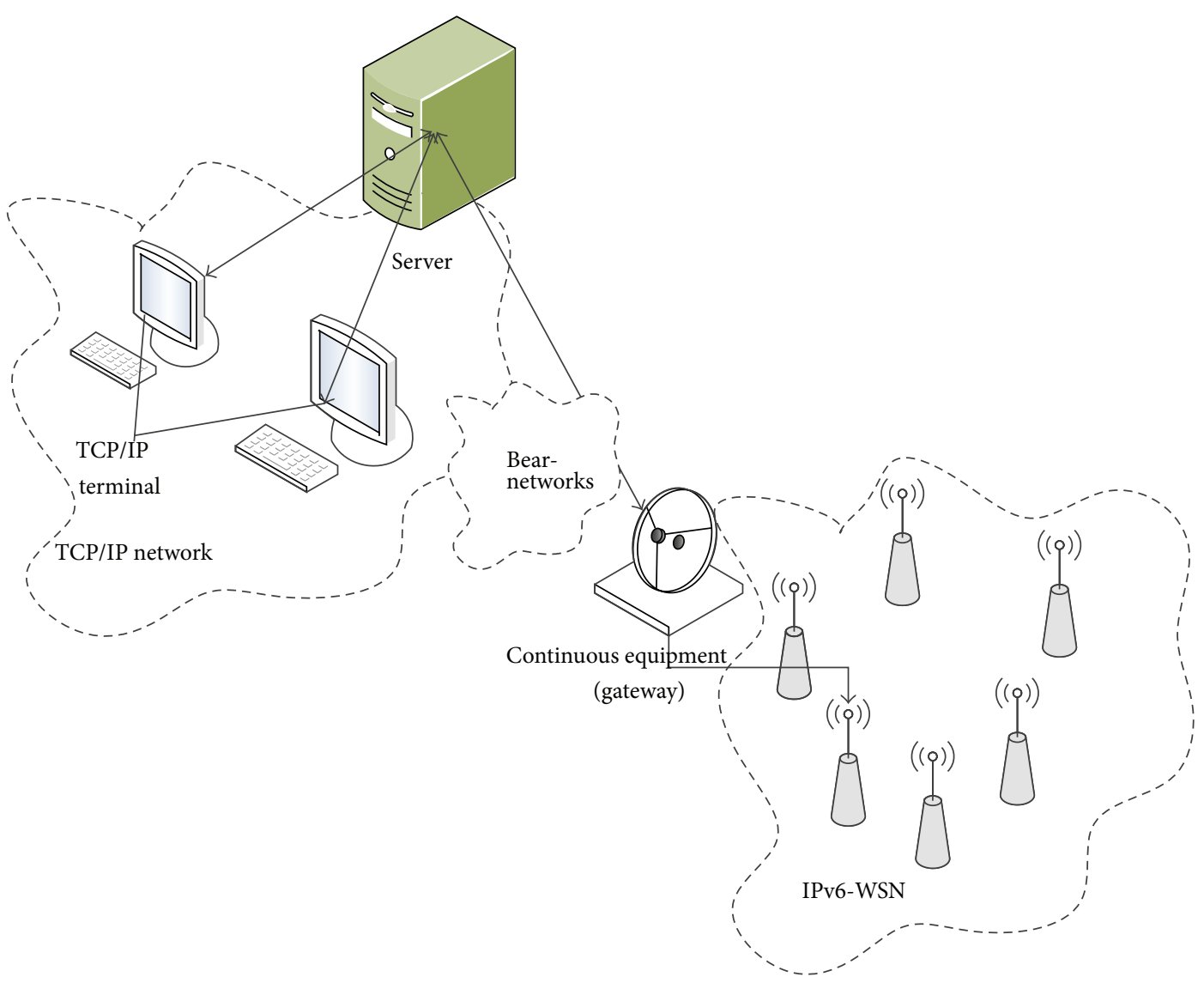

FIGURE 8: IPv6-WSN access existing network through a gateway device.

information. Users can access, control, and manage equipment effectively.

3.4. Internet Access. Sensor nodes have many access points to transfer data to the server-side through the gateway node, especially Ethernet access point and CDMA access point.

(a) Ethernet Access. Ethernet technology is currently one of the most widely used LAN technologies, as more than $70 \%$ of the network is based on the Ethernet protocol. Gateway device using Ethernet access is shown in Figure 9.

Gateway device adds Ethernet port and Ethernet frame control module in the central control module. The conversion is realized between Ethernet frame format and IEEE 802.15.4 frame format in the central control module in order to support IPv6-WSN to access Ethernet.

(b) GPRS/CDMA Access. GPRS systems provide data services on wireless systems through the introduction of packet data units in the existing GSM system. With the actual transfer rates up to $56 \mathrm{kbit} / \mathrm{s}$, they are particularly suitable for the transfer of small and frequent real-time data services. CDMA $\mathrm{lx}$ is a new bearer service developed based on CDMA IS.95. It is used to provide the data service in group and support for two-way symmetrical data transmission by $110 \mathrm{kbit} / \mathrm{s} \sim$ $120 \mathrm{kbit} / \mathrm{s}$. As a bearer network, GPRS/CMDA system with a port network structure allocates independent address for the user in order to achieve the end-to-end data application. Both access methods are basically similar as shown in Figure 10.

3.5. Protocol Design. In order to achieve the seamless connectivity of the network layer IPv6 and IEEE802.15.4 MAC layer, 6LoWPAN working group adds a network adaptation layer in the middle of MAC layer and network layer. The adaptation layer mainly completes header compression and the fragmentation during restructuring and routing of IP datagram. A WSN representative based on IP technology 6LoWPAN takes the difficult task of interconnectivity between WSN and a variety of IP-based networks and eventually manages them at the outset.

6LoWPAN defines a miniature TCP/IPv6 protocol stack for each sensor node, which makes a single node communicate like a PC application of IP technology. It not only achieves seamless interconnection between WSN and IP network but also ensures the end-to-end communication between nodes Internet. It is very important for WSN to collect remote data and control Internet nodes.

6LowPAN has its unique advantages that IPv6 can provide a large number of IP addresses. 6LoWPAN device is able to use the IPv6 address interoperably. For there is lack of support for IPv6, ZigBee network [25] could not access Internet. This research proposes the combination of the two protocols. 


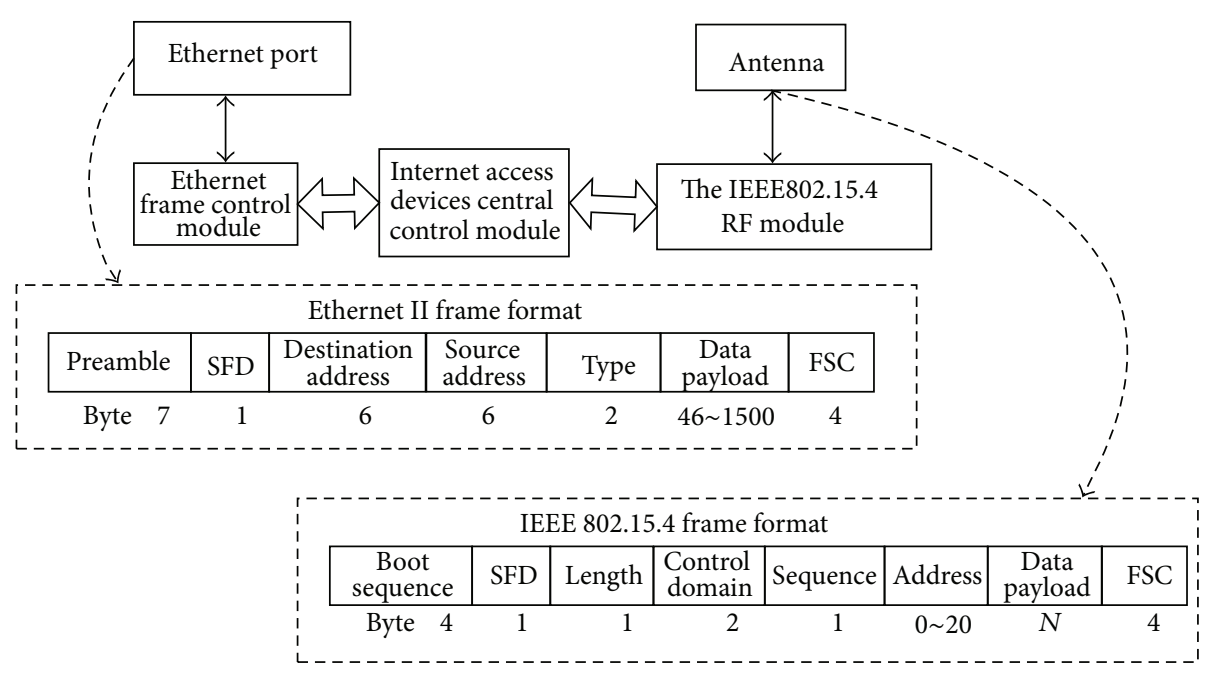

FIGURE 9: Ethernet access model.

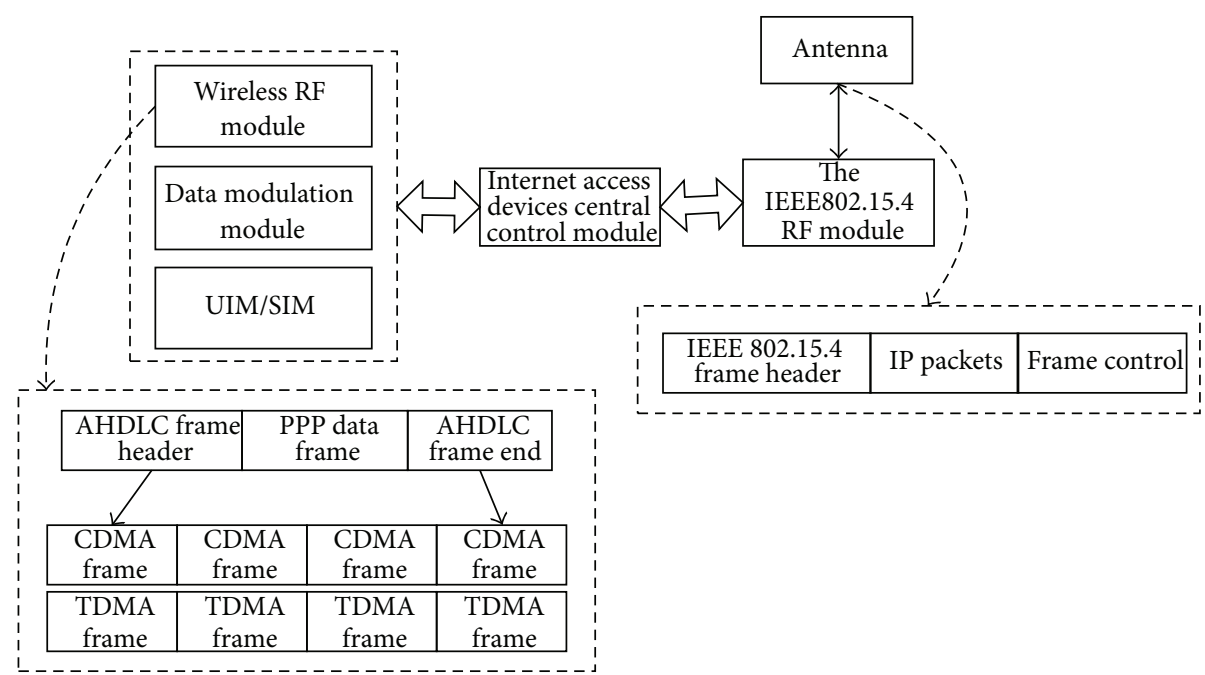

FIGURE 10: GPRS/CDMA access model.

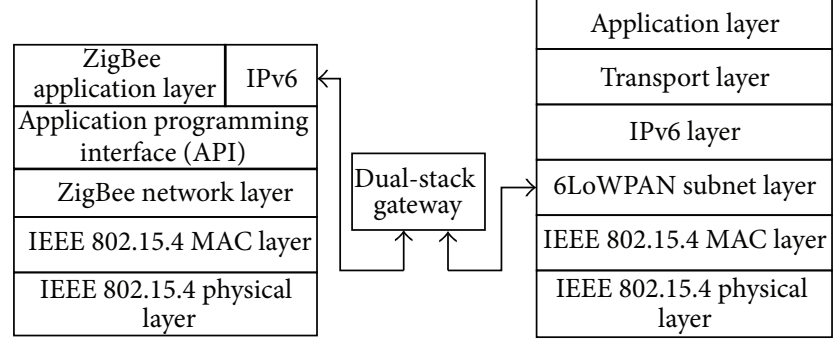

Figure 11: Dual-stack gateway of 6LowPan-ZigBee.

(a) Dual-Stack Gateway of 6LowPan-ZigBee. As shown in Figure 11, dual-stack gateway of 6LowPan-ZigBee is responsible for the conversion between the two data formats. All ZigBee nodes and 6LoWPAN nodes can communicate with each other through this dual-stack gateway. (b) Dual-Stack Node of 6LoWPAN-ZigBee. In order to communicate with IPv6, IPv6 protocol stack is set on the application layer of the ZigBee protocol stack. When dualstack node receives a packet, ZigBee protocol stack regards IPv6 data as a simple application layer data. When the packet arrives at the application layer, it removes the payload and assigns the control to IPv6 protocol stack. When dual-stack node sends packets, it also follows the principle regarding IPv6 data as a simple application layer data. So in this way we can achieve interoperability between these two types of nodes without using gateway or bridge to support ZigBee and 6LoWPAN protocol stack.

\section{The Simulation of ECIS}

In order to improve interconnection design, functions of 6LoWPAN adaptation layer in NS2 802.15.4 Simulator SSCS 
TABLE 1: Packet transmission success rate.

\begin{tabular}{lccc}
\hline & $\begin{array}{c}\text { Total number } \\
\text { of sent packets }\end{array}$ & $\begin{array}{c}\text { Total number of } \\
\text { received packets }\end{array}$ & $\begin{array}{c}\text { Packet transmission } \\
\text { success rate }\end{array}$ \\
\hline $\begin{array}{l}\text { Original } \\
\text { scheme }\end{array}$ & 4116 & 3271 & $79.47 \%$ \\
ECIS & 4116 & 3596 & $87.37 \%$ \\
\hline
\end{tabular}

layer were extended to complete the NS2 simulation of the interconnection system.

4.1. Simulation Scene Design. On the basis of existing modules in NS2, the simulation of following performance indicators is made by setting certain simulation scenario including packet transmission success rate, MAC layer data traffic, and average remaining energy as shown in Figure 13.

Here is the NS2 simulation scenario. IEEE 802.15.4 runs at wireless link of $2.5 \mathrm{GHz} / 250 \mathrm{kbps}$. $21 \mathrm{WSN}$ nodes are uniformly distributed in a $50 * 50 \mathrm{~m}^{2}$ area. The node transmitter range is set as $15 \mathrm{~m}$. NS2 run time is set as $100 \mathrm{~s}$. Application layer packet size is controlled within the 50 bytes. Radio propagation model is set as two-ray ground. Antenna type is set as Omni antenna. The routing is set as AODV. The trace tracks only the MAC layer. Initial energy is set as 5 Joule, and so forth.

According to the functional modules in NS2, we set specific simulation parameters, the time and type of sending packets to each node. Use AODV routing in NS2, write the ipv6.tel script, and then run the simulation model. The file automatic generation module after running the ipv6.tel contains each node in the network sending and receiving packets time, packet type, packet size, packet uid, node residual energy, source, destination address, and so on in the $100 \mathrm{~s}$ simulation time (trace MAC layer).

\subsection{Analysis of Simulation Data}

4.2.1. Packet Transmission Success Rate. Packet transmission success rate refers to the percentage of received packets by destination node and packets sent by the source node. It reflects the reliability of network transmission. The greater the success rate is, the higher the reliability is. In the process of packet transmission, header compression mechanism that reduces the size of the packet improves the success rate of packet transmission by reducing the number of packet fragmentations. Two interconnection schemes' packet transmission success rates are shown in Table 1.

Comparing with the TCP/IPv6 interconnection scheme, packet transmission success rate of the improved interconnection scheme has improved by $7.9 \%$. The introduction of IPv6 and UDP header compression mechanism in WSN reduces the size of the packet so that the number of packet fragmentations is drastically reduced. It also reduces transmission failure probability caused by packet loss or reorganization timeout during transmission. As a result, it improves packet transmission success rate (Figure 12).

\begin{tabular}{|c|c|}
\hline $\begin{array}{c}\text { ZigBee } \\
\text { application layer }\end{array}$ & IPv6 \\
\hline $\begin{array}{c}\text { Application programming } \\
\text { interface (API) }\end{array}$ & Application layer \\
\hline \multirow{2}{*}{ ZigBee network layer } & IPv6 layer \\
\cline { 2 - 2 } & 6LoWPAN subnet layer \\
\hline IEEE 802.15.4 MAC layer \\
\hline
\end{tabular}

Figure 12: Dual-stack node of 6LoWPAN-ZigBee.

4.2.2. MAC Layer Data Traffic. MAC layer data traffic refers to the total number of received data bytes in unit of time in the MAC layer. It shows network data flow and congestion level. Header compression reduces the size of packet, which reduces the MAC layer data traffic. Data comparison chart of the two programs is shown in Figure 14.

There is a different degree of volatility in two interconnection schemes of MAC layer data traffic. The two peaks at $10 \mathrm{~s}$ and $50 \mathrm{~s}$ are caused by the time when nodes send packets during simulation. Compared with TCP/IPv6 interconnection scheme, there are different degrees of reduction of IEEE802.15.4 MAC layer data traffic in improved interconnection scheme at any time. Curve is relatively stable. The gap between the two has always been maintained within a certain range, which shows that header compression mechanism has played a certain role in the reduction of the MAC layer data traffic. However, the limitations of header compression indicate that reduction of MAC layer data traffic is limited to a certain range.

4.2.3. Average Remaining Energy. The purpose of WSN design is lower power consumption. Header compression mechanism reduces the size of the packets. The incorporation of historical data query record reduces the node working hours among multiuser agent. They both play a role in the reduction of the energy consumption. The histogram of the remaining energy data change of WSN node in each measured moment is shown in Figure 15.

Average remaining energy of nodes in two schemes is onto a downward trend. Comparing with the original scheme, WSN nodes average remaining energy is lager and the decline is more slow at the same time in ECIS. This indicates that the ECIS fully meets the demand for WSN low-power design.

\section{Conclusion}

The research studies the interconnection scheme between WSN and Internet based on the 6LoWPAN. First, it designs an interconnection scheme between WSN and Internet based on the 6LoWPAN including structure design, interface design, Internet access, and protocol design. Secondly, it investigated the technology of 6LoWPAN energy control, 


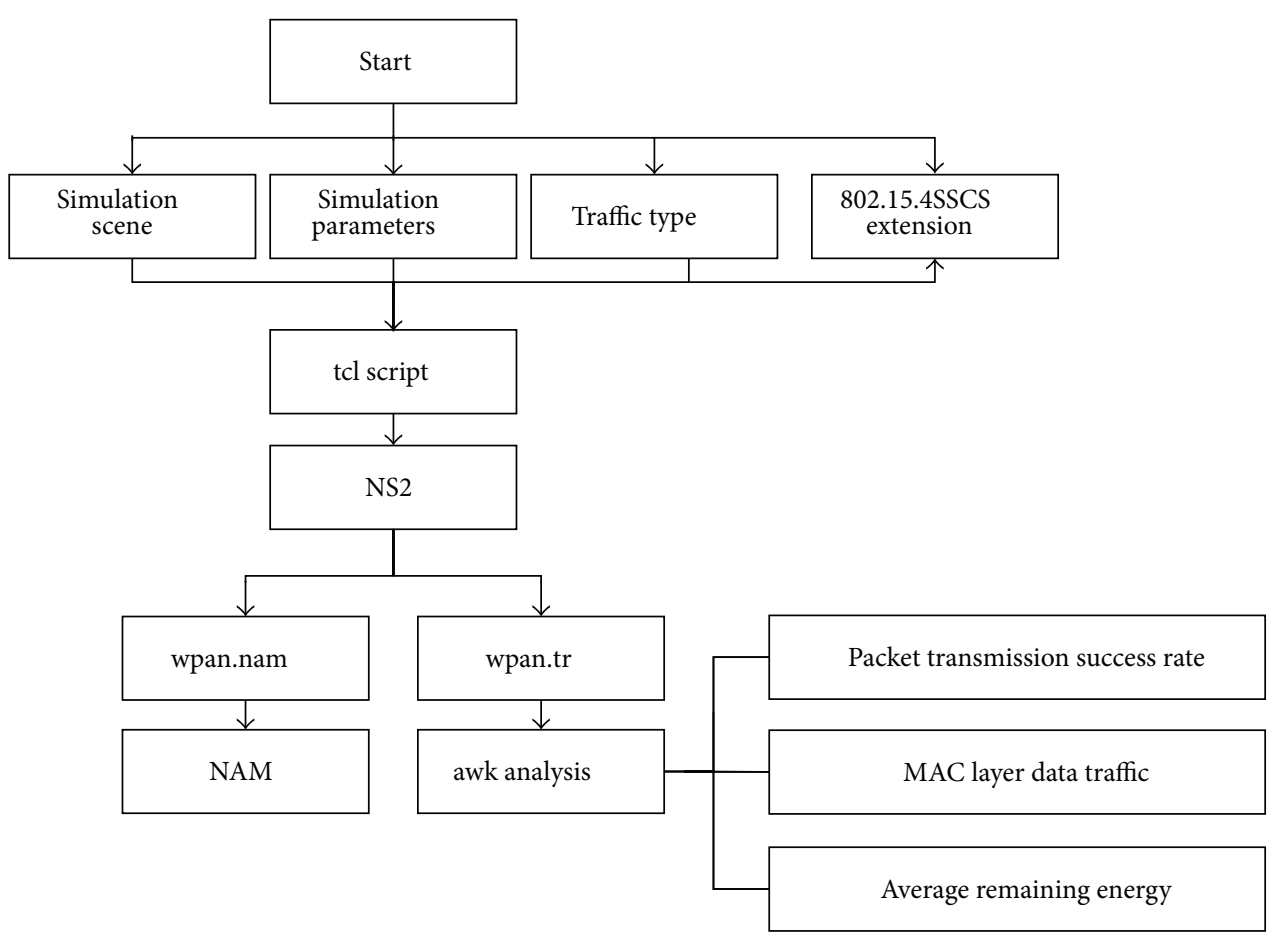

FIGURE 13: Simulation operational processes.

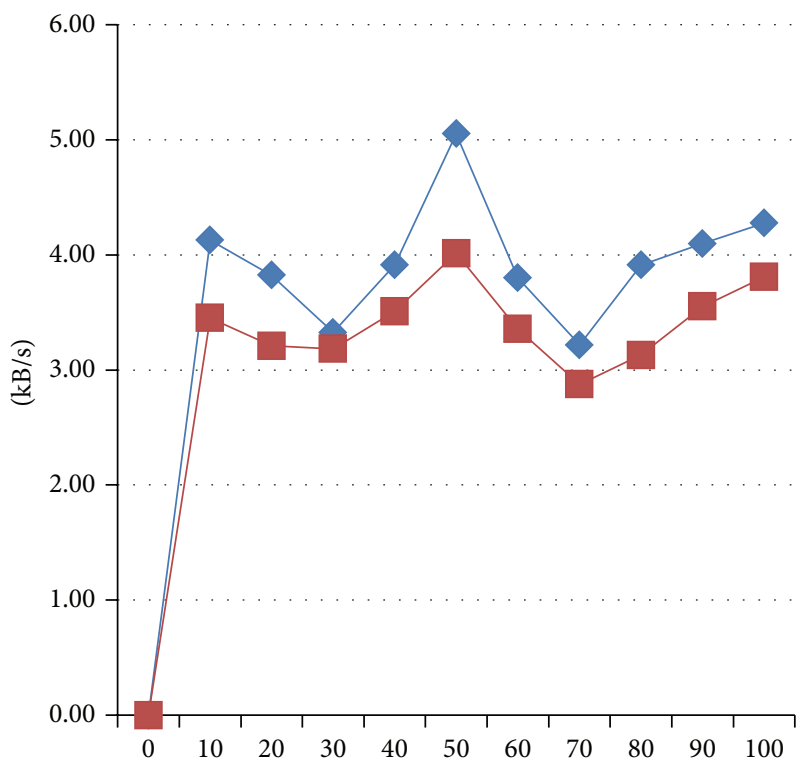

(s)

Original interconnection scheme

ECIS

FIGURE 14: Curve diagram of MAC layer data traffic.

and it proposed cross-layer energy-saving strategies in network interface layer, the adaptation layer, routing layer, and network layer. At last, the research shows the design and implementation of energy-saving dual-network supported

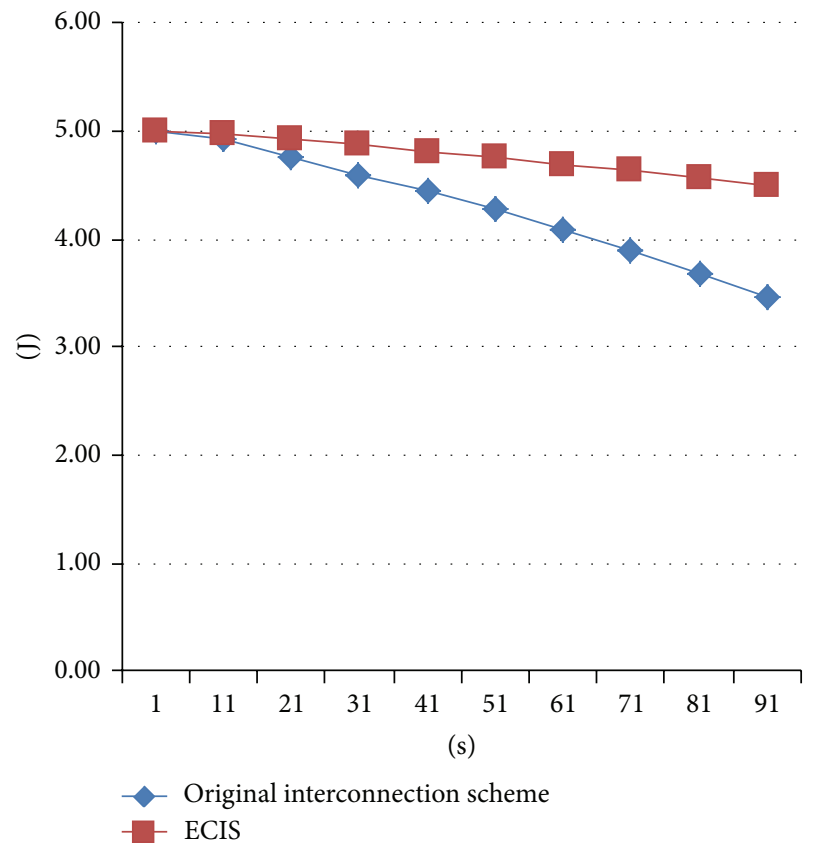

FIGURE 15: Average remaining energy data change of nodes.

by simulation experiment. Future work will be focusing on large-scale, dynamically changing WSN node in the actual hardware platform environment, which has significant practical impact in our society. 


\section{Conflict of Interests}

The authors declare that there is no conflict of interests regarding the publication of this paper.

\section{Acknowledgments}

This work is partially supported by a Beijing Natural Science Fund with Contract no. 4112044, a Ph.D. Programs Foundation Fund of Ministry of Education of China with Contract no. 20120009110009, and an EU 7th Framework Programme Fund with Contract no. EU IRSES 612546 ENRICH.

\section{References}

[1] L. Sun and J. Li, Wireless Sensor Networks, Tsinghua University Press, Beijing, China, 2005.

[2] N. Li and X. Huang, "A context system for 6LoWPAN network," in Proceedings of the 4th IEEE International Conference on Broadband Network and Multimedia Technology (IC-BNMT '11), pp. 522-525, IEEE, Shenzhen, China, October 2011.

[3] G. Mulligan, "The 6LoWPAN architecture," in Proceedings of the 4th Workshop on Embedded Networked Sensors (EmNets '07), pp. 78-82, June 2007.

[4] The Advanced Configuration \& Power Interface (ACPI) Specification-Revision 4.0, http://www.acpi.info/.

[5] C. E. Perkins and E. M. Royer, "Ad-hoc on-demand distance vector routing," in Proceedings of the 2nd IEEE Workshop on Mobile Computing Systems and Applications (WMCSA '99), pp. 90-100, February 1999.

[6] J. Baliga, R. Ayre, K. Hinton, and R. S. Tucker, "Photonic switching and the energy bottleneck," in Proceedings of the Photonics in Switching, pp. 125-126, IEEE, August 2007.

[7] R. Tucker, "Will optical replace electronic packet switching?" Optoelectronics \& Communications, 2007.

[8] L. G. Roberts, “A radical new router," IEEE Spectrum, vol. 46, no. 7, pp. 34-39, 2009.

[9] M. Baldi and Y. Ofek, "Time for a 'greener' internet," in Proceedings of the IEEE International Conference on Communications Workshops (ICC '09), pp. 1-6, June 2009.

[10] M. Zhang, C. Yi, B. Liu, and B. Zhang, "GreenTE: Power-aware traffic engineering," in Proceedings of the 18th IEEE International Conference on Network Protocols (ICNP '10), pp. 21-30, IEEE, October 2010.

[11] L. Chiaraviglio, M. Mellia, and F. Neri, "Energy-aware backbone networks: a case study," in Proceedings of the IEEE International Conference on Communications Workshops (ICC '09), pp. 1-5, June 2009.

[12] D. Harris, "Sponsored editorial-DESIGN FAQs-reducing FPGA power consumption," Electronic Design, vol. 56, no. 3, p. 22, 2008.

[13] J. Noguera and I. O. Kennedy, "Power reduction in network equipment through adaptive partial reconfiguration," in Proceedings of the International Conference on Field Programmable Logic and Applications (FPL '07), pp. 240-245, August 2007.

[14] M. Gupta and S. Singh, "Greening of the internet," in Proceedings of the Conference on Applications, Technologies, Architectures, and Protocols for Computer Communications, pp. 19-26, ACM, Karlsruhe, Germany, August 2003.

[15] A. Cianfrani, V. Eramo, M. Listanti, M. Marazza, and E. Vittorini, "An energy saving routing algorithm for a green OSPF protocol," in Proceedings of the INFOCOM IEEE Conference on Computer Communications Workshops, pp. 1-5, IEEE, San Diego, Calif, USA, March 2010.

[16] S. Ricciardi, D. Careglio, G. Santos-Boada, J. Solé-Pareta, U. Fiore, and F. Palmieri, "Towards an energy-aware Internet: modeling a cross-layer optimization approach," Telecommunication Systems, vol. 52, no. 2, pp. 1247-1268, 2013.

[17] R. Hua, The Design of a Light-Weight TCP/IP Protocol Based on Wireless Sensor Network Application, Beijing Jiaotong University, Beijing, China, 2011.

[18] H. Dai and R. Han, "Unifying micro sensor networks with the Internet via overlay networking," in Proceedings of the 29th Annual IEEE Conference on Local Computer Networks, Tampa, Fla, USA, November 2004.

[19] Q. Ge, "Memory management strategies on TCP/IP performance for embedded application," in Proceedings of the 2nd International Symposium on Intelligent Information Technology Application (IITA '08), vol. 2, pp. 770-774, Shanghai, China, December 2008.

[20] X. Zhao, X. Wang, W. Yu, and X. Zhou, "An efficient broadcast authentication protocol in wireless sensor networks," Chinese Journal of Electronics, vol. 18, no. 2, pp. 368-372, 2009.

[21] J.-G. Ko, Y.-H. Cho, and H. Kim, "Performance evaluation of IEEE 802.15.4 MAC with different backoff ranges in wireless sensor networks," in Proceedings of the 10th IEEE Singapore International Conference on Communications Systems (ICCS '06), pp. 1-5, October 2006.

[22] T. Hu, X. Cui, and Y. Yao, "A new interconnection scheme for WSN and IPv6-based internet," in Proceedings of the IEEE Youth Conference on Information, Computing and Telecommunication (YC-ICT '09), pp. 34-37, IEEE, Beijing, China, September 2009.

[23] A. Lahmadi, C. Brandin, and O. Festor, "A testing framework for discovering vulnerabilities in 6LoWPAN networks," in Proceedings of the 8th IEEE International Conference on Distributed Computing in Sensor Systems (DCOSS '12), pp. 335-340, May 2012.

[24] Y. Zhang, P. Xu, G. Bi, and F. S. Bao, "Analysis of energy efficiency and power saving in IEEE 802.15.4," in Proceedings of the IEEE Wireless Communications and Networking Conference (WCNC '07), pp. 3330-3334, IEEE, March 2007.

[25] 2013, http://www.zigbee.org/. 

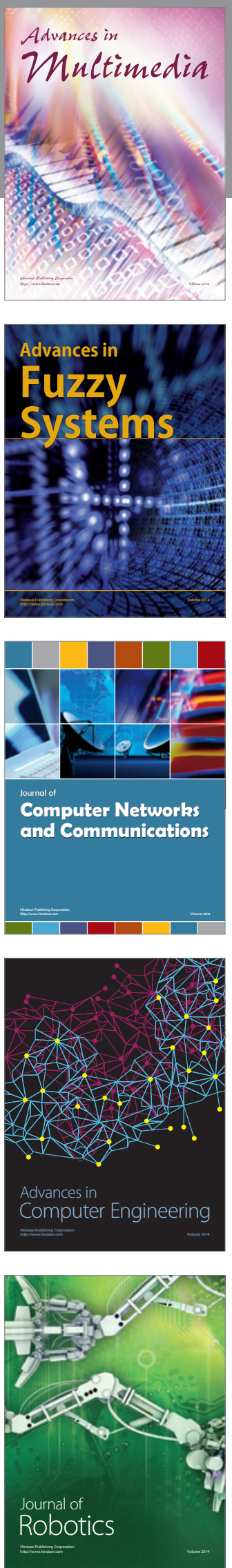

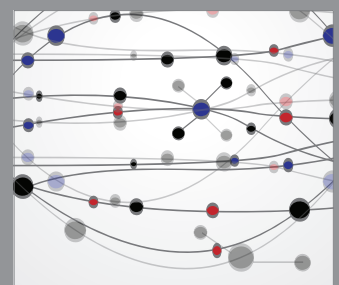

The Scientific World Journal
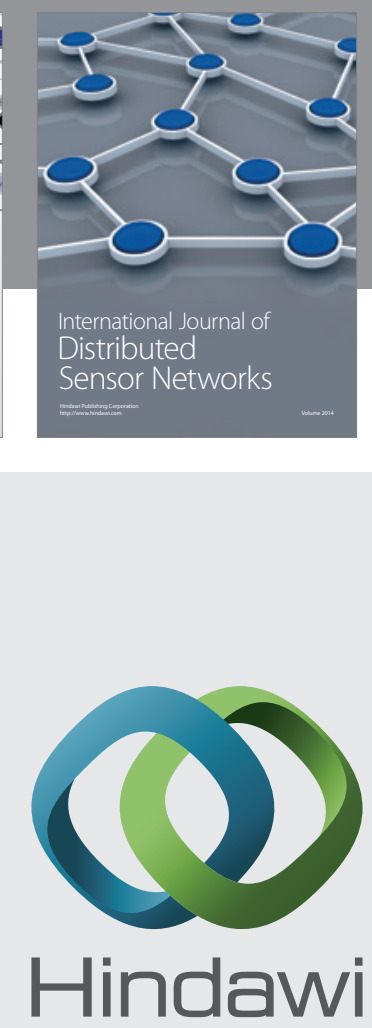

Submit your manuscripts at

http://www.hindawi.com
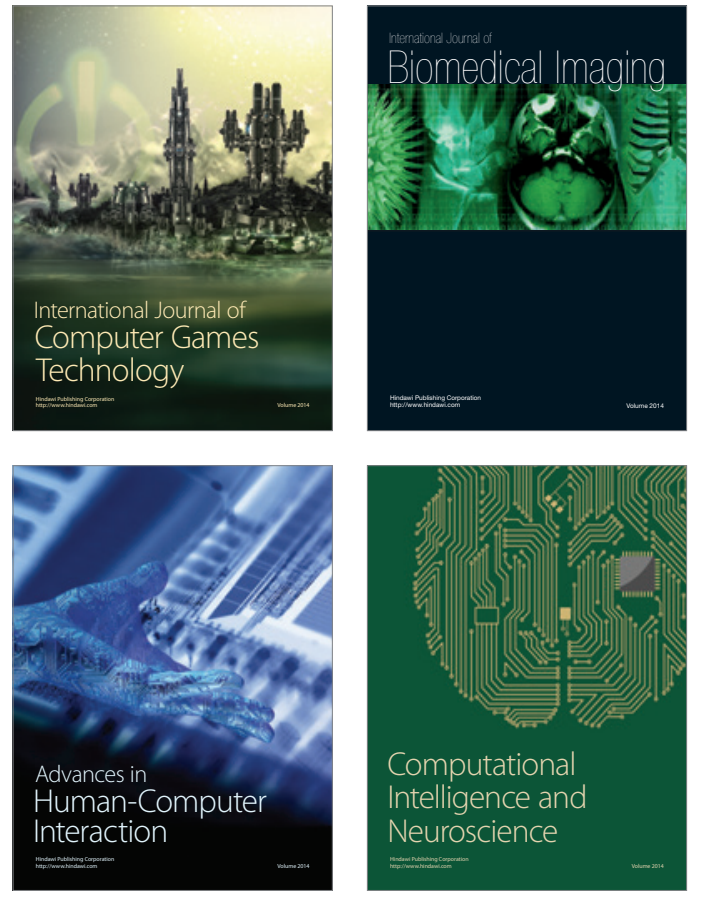
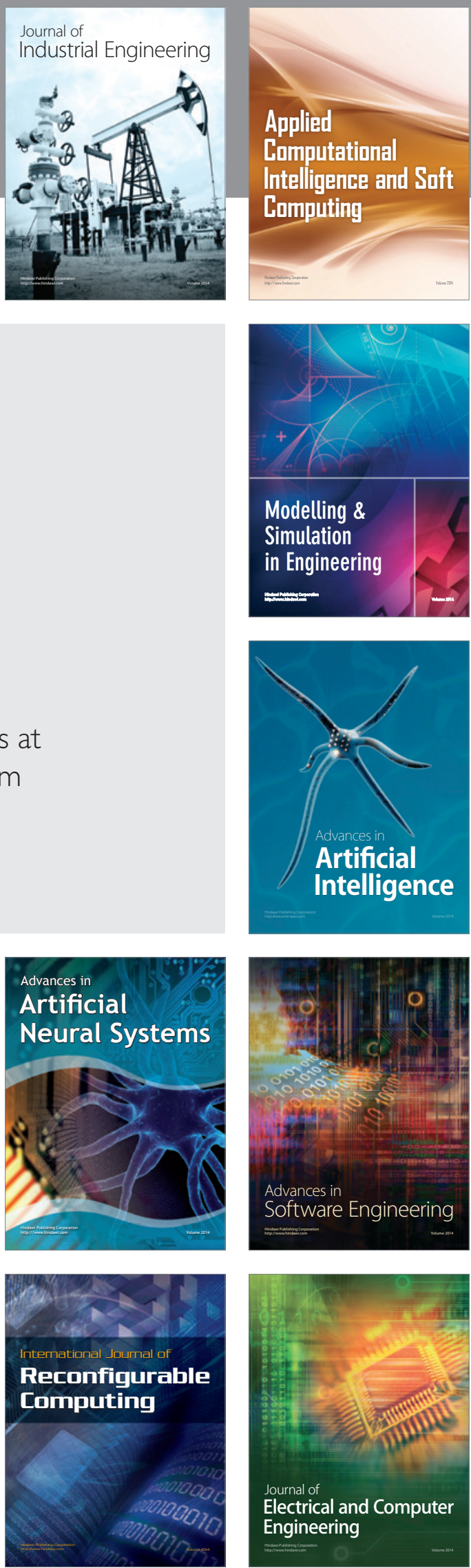